\title{
Fungal monopoly promotes allergy
}

\section{antibiotics}

can promote allergic airway inflammation by leading to the overgrowth of commensal fungi in the gut
The intestinal microbiota shapes the nature of the host immune system, and imbalances in the composition of the microbiota have been associated with both intestinal and extraintestinal disease. Kim et al. now show that the treatment of mice with antibiotics can promote allergic airway inflammation by leading to the overgrowth of commensal fungi in the gut.

Several studies have shown how commensal bacteria can influence systemic immune responses, but the effects of other commensal microorganisms are poorly understood. The authors treated mice with antibiotics to induce dysbiosis and found that this increased the susceptibility of the animals to allergic airway inflammation induced by papain or house dust mite extract. Compared with controls, antibiotic-treated mice showed enhanced

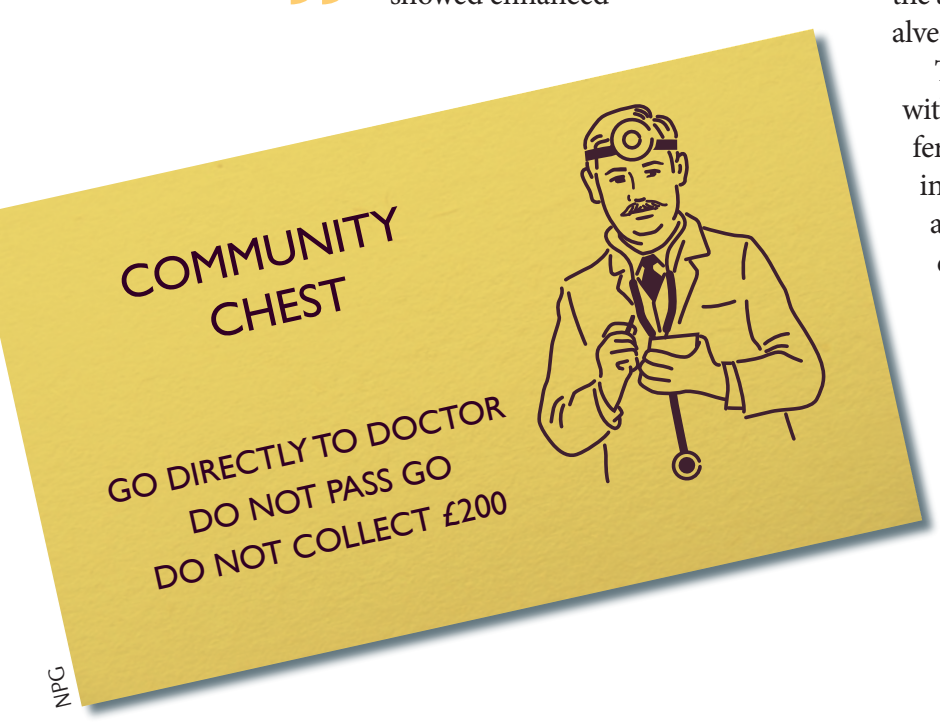

production of type 2 cytokines and chemokines, increased inflammatory cell infiltration and higher levels of goblet cell hyperplasia in response to airway allergens.

The antibiotic-mediated alteration of alveolar macrophage function seemed to contribute to the increase in airway inflammation, as untreated animals that received alveolar macrophages from antibiotic-treated mice developed more severe airway inflammation than animals that received alveolar macrophages from control mice. Notably, alveolar macrophages from antibiotic-treated mice showed an increase in the expression levels of genes that are associated with an alternatively activated M2 phenotype, such as Arg1, Chi3l3 and Retnla. This suggested that antibiotic treatment was increasing susceptibility to allergic airway disease by promoting the acquisition of an M2 phenotype in alveolar macrophages - but how?

The authors found that treatment with various antibiotics led to different degrees of fungal outgrowth in the intestine. Remarkably, the antibiotics that promoted a greater extent of fungal outgrowth in the gut also promoted increased levels of inflammatory cell infiltration in response to airway allergens. The authors isolated a fungal strain, Candida parapsilosis, from the faeces of antibiotic-treated mice and showed that transferring C. parapsilosis to mice that did not carry this strain increased their susceptibility to allergic airway inflammation. Oral treatment of mice with Candida species isolated from humans also led to fungal outgrowth in the gut and exacerbated allergic airway inflammation.

Fungal outgrowth in the gut led to increased serum levels of prostaglandin E2 (PGE2), which promoted the development of M2 macrophages. Interestingly, C. parapsilosis was shown to directly convert exogenous arachidonic acid into prostaglandins. Finally, the authors showed that treating mice with aspirin - an inhibitor of prostaglandin synthesis - led to a reduction in M2 macrophage differentiation and also to decreased allergic airway inflammation.

Of note, previous studies have reported that patients with allergic disease show fungal overgrowth in the gut. This study suggests that such intestinal dybiosis could be a causative factor in the development of allergic disease.

Yvonne Bordon

ORIGINAL RESEARCH PAPER Kim, Y.-G. et al.

Gut dysbiosis promotes M2 macrophage polarization and allergic airway inflammation via fungi-induced PGE. dx.doi.org/10.1016/j.chom.2013.12.010 (2014) 